\title{
Estudo de Topologias Híbridas em Redes Ópticas Passivas
}

\author{
Tany V. Villalba, Mônica L. Rocha, Murilo A. Romero, Sandro M. Rossi, Marcos P. Mokarzel, \\ Marcos R. Salvador e Miriam R. X. Barros
}

\begin{abstract}
Resumo - Este trabalho apresenta um estudo de três topologias de redes ópticas passivas, barramento, árvore e anel, com características híbridas de distribuição de potência (simétrica e assimétrica). Comparada à árvore simétrica, convencionalmente usada, a topologia em anel com distribuição assimétrica pode levar a uma melhoria de $3 \mathrm{~dB}$ no orçamento de potência em configurações de rede onde os distribuidores estejam alinhados, por exemplo, ao longo de uma rodovia.
\end{abstract}

Palavras-Chave-Redes de acesso, Rede óptica passiva, rede de distribuição óptica.

Abstract-This article presents a study of three passive optical network topogies, bus, tree and ring, with hybrid power distribution (symmetric and asymmetric). In comparison to the conventional symmetric tree, the asymmetric ring has lead to a $3 \mathrm{~dB}$ improvement, in terms or power budget. That result applies when the splitters are aligned - as it happens along a road.

Index Terms-Access networks, Optical passive network, optical distribution network.

\section{INTRODUCTION}

$\mathrm{O}$ aumento crescente da oferta de serviços e aplicações, principalmente aqueles envolvendo transmissão de imagem (por exemplo, vídeo-conferência, vídeo sob demanda e jogos on-line), estimula e justifica a implantação de redes ópticas de acesso. Neste cenário, a utilização de fibras ópticas nas premissas do usuário já permite, a um custo competitivo, que se disponibilize para cada cliente uma capacidade de transmissão elevada (até $2,5 \mathrm{~Gb} / \mathrm{s}$ por até algumas dezenas de quilômetros), tanto na descida quanto na subida dos dados. Além disso, comparadas às redes de acesso convencionais, estas redes, mais conhecidas como redes ópticas passivas (Passive Optical Network, PON), apresentam facilidade de instalação e atualização, baixo custo de operação e manutenção, confiabilidade, imunidade eletromagnética e cabos mais leves e compactos [1].

A maioria das redes PON sendo instaladas no mundo têm arquitetura do tipo ponto-multiponto com um ou mais níveis de derivação de potência, via acopladores ópticos passivos, para distribuição do sinal. O acesso ao meio na transmissão ascendente é feito através de multiplexação no tempo (TDMA), para evitar colisões no acoplador. Das duas tecnologias PON em implantação (Gigabit PON, GPON, padronizada pelo ITU-T G.984 [2]-[5], e Ethernet PON, EPON, padronizada pelo IEEE 802.3ah [6]), o GPON,

Este trabalho é financiado pela Fundo para o Desenvolvimento Tecnológico das Telecomunicações.

T. V. Villalba, M. L. Rocha e M. A. Romero são da Escola de Engenharia de São Carlos, EESC, Universidade de São Paulo (tanyvillalba@yahoo.com).

S.M. Rossi, M.P. Mokarzel e M.R. Salvador são da Fundação CPqD. (sandro@cpqd.com.br). M. R. X. Barros (miriam.barros@gmail.com). adotado no Brasil, oferece vantagens como taxas de tráfego ascendente e descendente mais altas, maior eficiência de banda (na relação cabeçalho/carga útil) e maior variedade de serviços. Pelo lado do EPON, destaca-se positivamente seu custo mais baixo e a maior maturidade tecnológica.

Uma dos desafios de planejamento e projeto de uma rede PON diz respeito à distribuição dos divisores de potência, em termos de posicionamento e razão de divisão. Alguns fatores que influenciam na escolha da topologia são: distribuição geográfica dos usuários (determinará o número e o posicionamento dos divisores ópticos) e a quantidade e comprimento de cabos ópticos que farão a ligação entre uma OLT (Optical Line Terminal, localizada na Central) e cada uma das ONUs (Optical Network Units, localizadas nas premissas do usuário).

Uma análise realizada em redes de acesso instaladas mostrou que muitas das fibras utilizadas não são necessárias [7], [8]. Isso ocorre porque não houve nenhum estudo e otimização preliminar, ou seja, o cabeamento ocorreu enquanto houve necessidade dos usuários. Esse fato teve como conseqüência o crescimento desordenado e não planejado da rede, planejamento no qual uma limitação importante a ser levada em conta é o alcance das redes ópticas, que pode ser de ordem física e lógica. No caso da primeira, o alcance depende fortemente da topologia e distribuição dos elementos ópticos que, além das perdas pela divisão de potência, podem sofrer de efeitos como atenuação nos comprimentos de ondas utilizados, elevado número de ONUs ligadas a cada OLT e baixa sensibilidade dos receptores. A limitação lógica está relacionada aos protocolos de comunicação entre OLT e ONUs, que tem como requisito uma janela temporal máxima para recebimento de mensagens.

Em trabalho anterior [9], visando otimizar o alcance geográfico de uma rede PON (i.e. a máxima distância entre a OLT e a ONU mais distante) propusemos o uso de uma distribuição híbrida simétrica e assimétrica da potência óptica na topologia barramento, adequada a uma distribuição linear de ONUs, por exemplo, uma demanda de tráfego ao longo de uma rodovia. A topologia proposta naquele trabalho, aplicável a barramentos lineares, tinha dois níveis de derivação, onde o primeiro era assimétrico (acopladores 1:2 usados para derivar uma fração da potência) e o segundo nível simétrico (acopladores 1:N). Avançando esta análise para um caso mais genérico, apresentamos neste artigo um estudo semelhante aplicado a três topologias físicas com um nível de derivação: barramento, árvore e anel (Fig. 1).

O artigo está assim estruturado: na seção II as topologias estudadas são descritas, bem como o formalismo matemático empregado na análise. Na seção III apresentamos os 
resultados obtidos para as combinações de distribuição e na seções IV e V, as discussões e conclusões do trabalho, respectivamente.

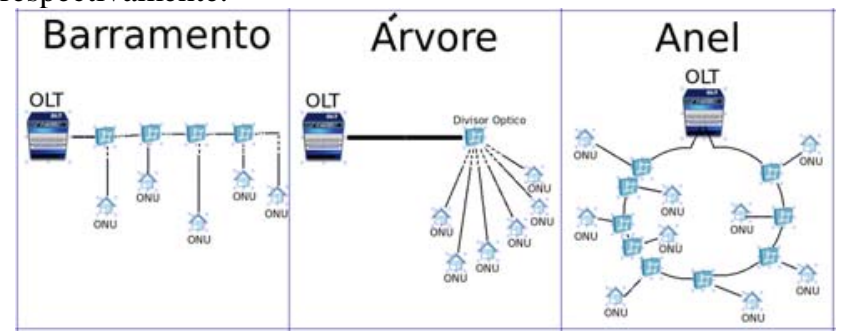

Fig. 1. Topologias analisadas: barramento, árvore e anel.

\section{TOPOlOGIAS DE DistRIBUIÇÃO}

Para analisar as três topologias mencionadas consideraremos inicialmente um único cenário físico, com apenas um nível de derivação. Posteriormente, para efeitos de comparação, fixaremos o posicionamento da OLT e das ONUs, encontraremos a metragem de cabos ópticos necessária e calcularemos o orçamento de potência para cada topologia. A distribuição física das três topologias é ilustrada na Fig. 2. Como no estudo anterior [6], o exemplo poderia ser aplicável a uma rede linear ao longo de uma rodovia.

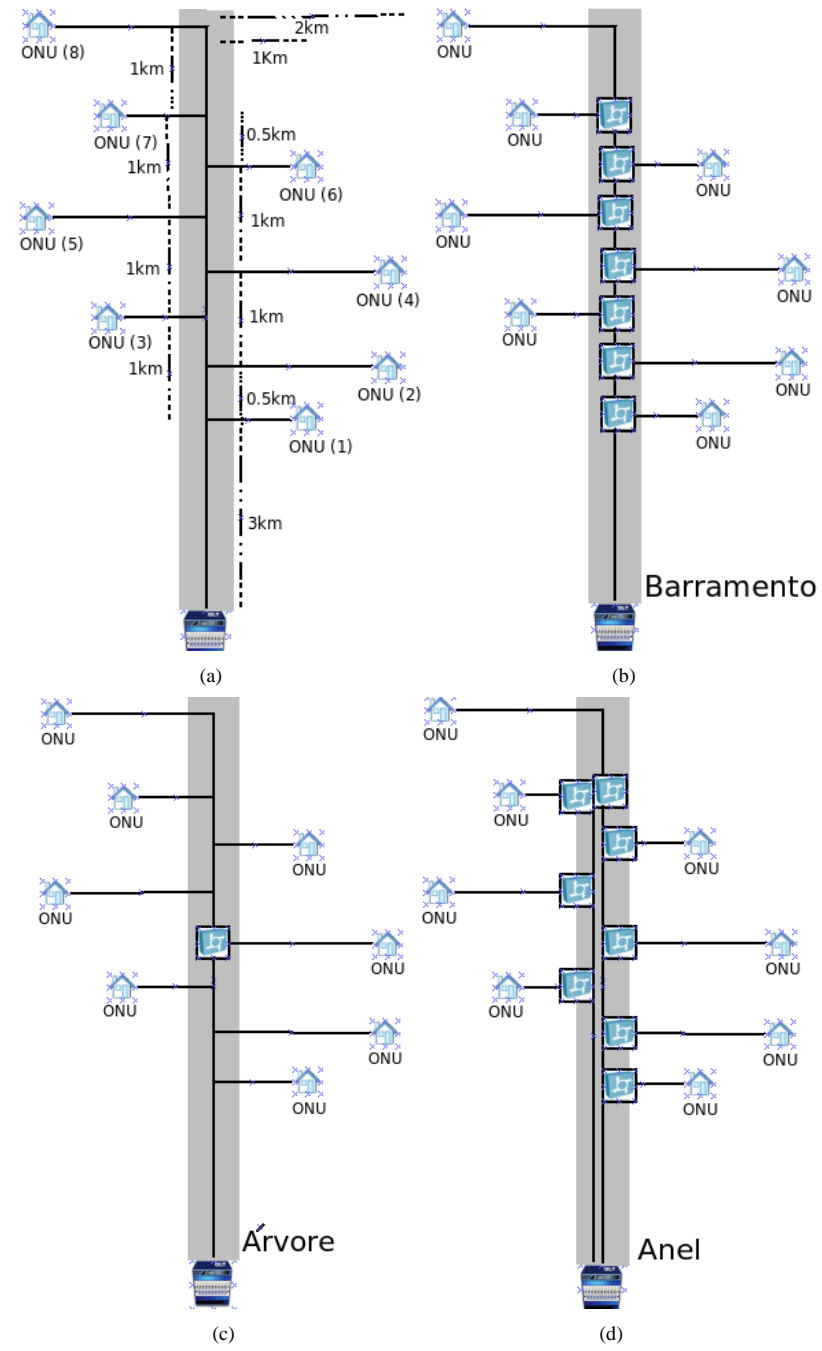

Fig. 2. Distribuições físicas com um estágio de derivação: (a) padrão de distâncias adotado; (b) barramento; (c) árvore e (c) anel.

Assumimos que as perdas da fibra para os sinais descendente $(1490 \mathrm{~nm})$ e ascendente $(1310 \mathrm{~nm})$ são 0,25 e $0,4 \mathrm{~dB} / \mathrm{km}$, respectivamente. Por esta razão, as análises são feitas somente para o tráfego ascendente, pois as limitações por atenuação de pior caso estão associadas a ele. A potência óptica transmitida pela ONU é $+2 \mathrm{dBm}$ (valor mínimo especificado para transmissores na recomendação G.984.2). A perda do distribuidor simétrico 1:N é dada por [10 log $(1 / \mathrm{N})]$. A perda de conexão de cada porta dos acopladores é considerada igual a $0,2 \mathrm{~dB}$.

Para avaliar estas redes em termos de orçamento de potência, a perda total do sinal, $\mathrm{P}_{\mathrm{T}}$, entre a OLT e uma ONU, é dada por:

$$
P_{T}=2 P_{C}+P_{D, K}+2 k P_{C}+\sum_{i=1}^{k-1}\left(P_{T_{i}}+\alpha_{1310} l_{i}\right)
$$

onde $\mathrm{P}_{\mathrm{C}}$ é a perda por conexão óptica, $\mathrm{P}_{\mathrm{D}, \mathrm{K}}$ é a perda na derivação do k-ésimo divisor, $\mathrm{P}_{\mathrm{Ti}}$ é a perda na transmissão pelo i-ésimo divisor, $\alpha_{1310}$ é a atenuação da fibra, em $\mathrm{dB} / \mathrm{km}$, e $\mathrm{l}_{\mathrm{i}}$ é a distância entre o i-ésimo divisor e o anterior. As perdas são dadas em $\mathrm{dB}$ e os comprimentos em $\mathrm{km}$.

A margem de potência óptica, $\mathrm{M}$, em $\mathrm{dB}$, corresponde à uma reserva do orçamento de potência, para cobrir perdas não previstas no projeto sistêmico. No nosso caso, é dada por:

$M=P_{T x, O N U}-P_{T}-S_{R x, O L T}$

onde $\mathrm{P}_{\mathrm{Tx}, \mathrm{ONU}}$ é a potência óptica transmitida pela $\mathrm{ONU}$, em $\mathrm{dBm}$, e $\mathrm{S}_{\mathrm{Rx}, \mathrm{OLT}}$ é a sensibilidade do receptor da OLT, em dBm.

Seguindo o padrão mostrado na Fig. 2a, a Fig. 3 ilustra as distâncias entre a OLT e cada uma das ONUs para cada uma das topologias estudadas, distâncias que utilizamos para o cálculo do orçamento de potência. A Tabela 1, associada à Fig. 4 (número de divisores e comprimento total dos cabos ópticos, para cada topologia), sumariza os resultados obtidos. Percebe-se que as topologias em anel e barramento apresentam a mesma distância entre as ONUs e a OLT, apesar da primeira requerer cabeamento mais longo que a segunda. Isso é conseqüência do cabo duplo utilizado.

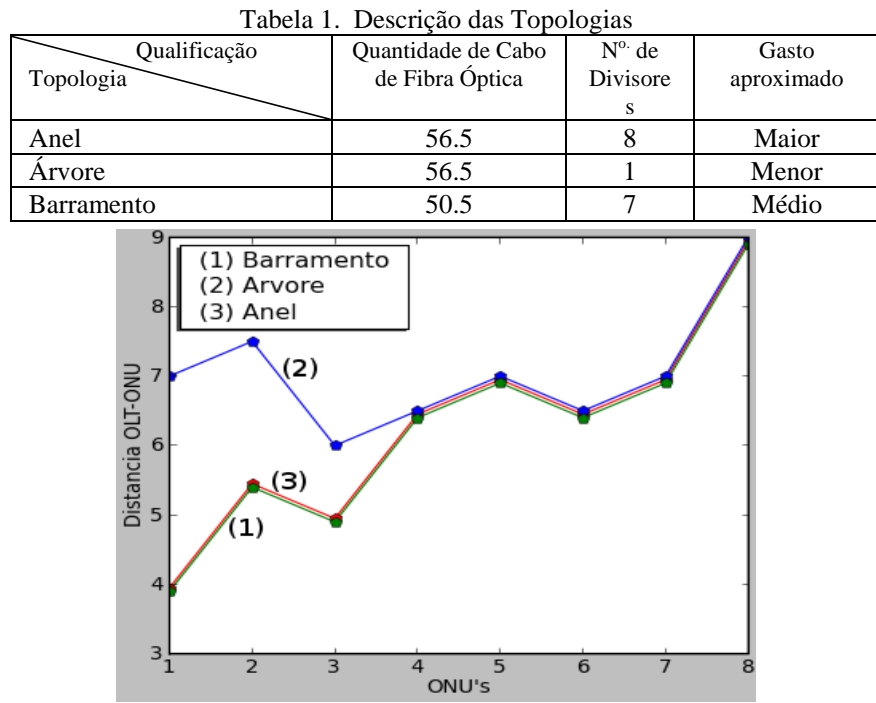

Fig. 3. Distância entre a OLT e cada ONU. 


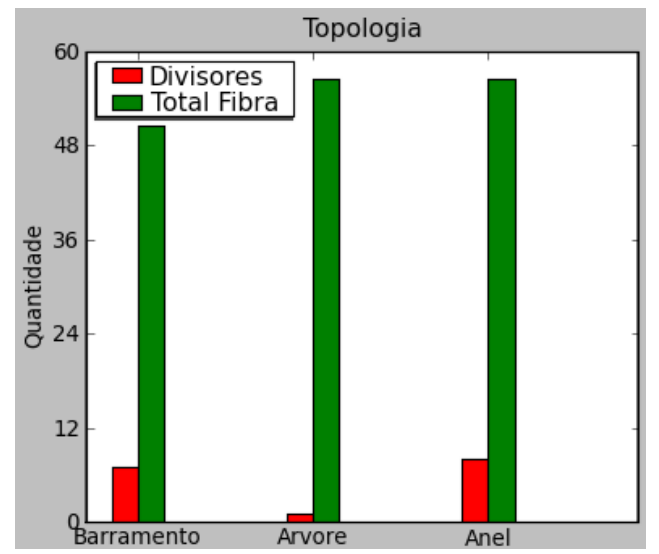

Fig. 4. Número de divisores e comprimento total cabos ópticos por topologia.

\section{Resultados}

Apresentamos na Fig. 5 os valores de margem de potência para cada ONU, simulados para todas as topologias escolhidas, utilizando inicialmente apenas divisores simétricos.

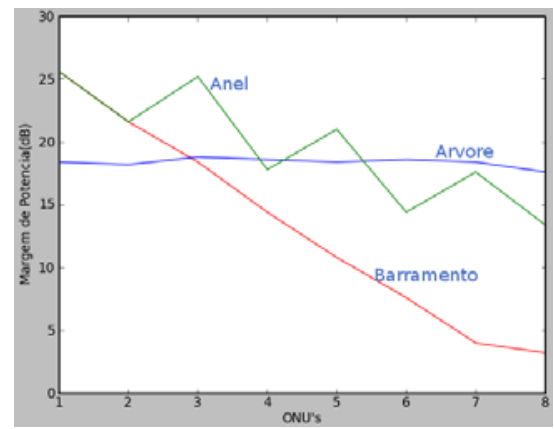

Fig. 5. Orçamento de potência para as topologias, usando apenas divisores simétricos.

Pode-se verificar que a curva para a topologia em árvore apresenta a melhor e mais homogênea margem de potência para o cenário escolhido, enquanto a topologia em barramento apresentou um decréscimo significativo à medida que um número mais expressivo de ONUs é percorrido. Por esta razão, os divisores com fração de derivação (“taps”) podem ser utilizados em topologias em anel e barramento para balancear a potência e melhorar o desempenho do sistema. Em nosso trabalho, denotamos por divisão assimétrica aquela que utiliza um acoplador 1:2 para derivar uma fração da potência óptica e o uso combinado de divisores simétricos e assimétricos resulta em topologias híbridas, em termos de divisão de potência. Para comparação utilizaremos divisores assimétricos nas topologias anel e barramento, com os valores de divisão indicados na Tabela 2 (as porcentagens são obtidas por aproximação e correspondem à fração da potência que é derivada no nó) .

Tabela 2. Porcentagem de derivação simétrica progressiva empregada nos cálculos

\begin{tabular}{|l|c|c|}
\hline & Barramento \% Derivação & Anel \% Derivação \\
\hline DIV. ONU 1 & $7 \%$ & $18 \%$ \\
\hline DIV. ONU 2 & $10 \%$ & $27 \%$ \\
\hline DIV. ONU 3 & $11 \%$ & $17 \%$ \\
\hline DIV. ONU 4 & $16 \%$ & $45 \%$ \\
\hline DIV. ONU 5 & $21 \%$ & $30 \%$ \\
\hline DIV. ONU 6 & $29 \%$ & $100 \%$ \\
\hline
\end{tabular}

\begin{tabular}{|l|c|c|}
\hline DIV. ONU 7 & $47 \%$ & $43 \%$ \\
\hline DIV. ONU 8 & & $100 \%$ \\
\hline
\end{tabular}

Neste caso, como indicado na Fig. 6, a topologia em anel com divisores assimétrico apresenta a melhor resposta, seguida da topologia em árvore e barramento com divisores assimétricos. A Tabela 3 resume os resultados obtidos.

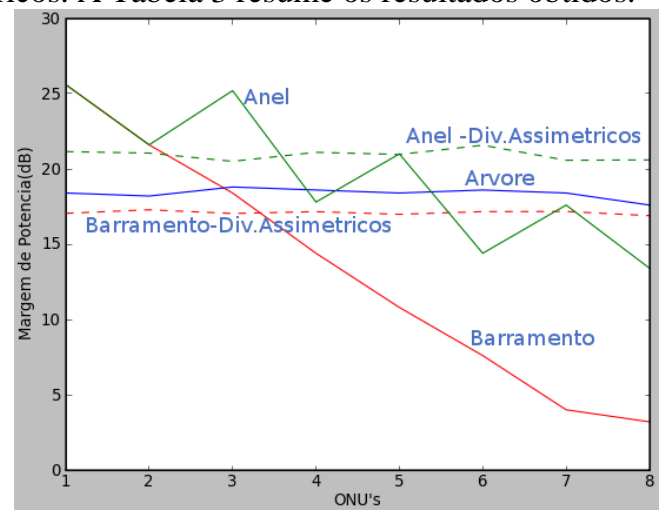

Fig. 6. Orçamento de potência para as topologias, usando também divisores assimétricos.

Tabela 3. Margens de Potência

\begin{tabular}{|l|c|c|c|}
\hline Topologia & $\begin{array}{c}\text { Média da } \\
\text { Margem de } \\
\text { Potencia (dB) }\end{array}$ & $\begin{array}{c}\text { Margem } \\
\text { Mínima de } \\
\text { Potência } \\
(\mathrm{dB})\end{array}$ & $\begin{array}{c}\text { Margem } \\
\text { Máxima de } \\
\text { Potência (dB) }\end{array}$ \\
\hline Anel & 19,5 & 13,4 & 25,6 \\
\hline Árvore & 18,3 & 17,6 & 18,8 \\
\hline Barramento & 13,2 & 3,2 & 25,6 \\
\hline Anel Div. Assimétrico & 21,5 & 20,5 & 20,9 \\
\hline Barramento Div. Assimétrico & 17,0 & 16,8 & 17,3 \\
\hline
\end{tabular}

\section{DISCUSSÃO}

Em aplicações onde várias ONUs estiverem dispostas de forma linear, a combinação de divisores assimétricos e simétricos pode reduzir a quantidade total de fibra necessária à cobertura da área, em comparação às topologias em árvore convencionais. Neste trabalho, estendemos o resultado obtido em [9] com barramento assimétrico, para topologias em árvore e anel assimétricos, constituindo uma categoria de redes ópticas passivas híbridas, em termos de divisão de potência. Embora nossos resultados não possam ser usados em generalizações, pois correspondem a uma amostragem relativamente pequena, podemos resumir as vantagens e desvantagens encontradas com os vários testes realizados para cada topologia:

- Topologia em anel: A principal motivação para o uso desta topologia é a possibilidade de proteção em cenários onde a continuidade da comunicação em caso de falhas é muito importante. No caso do exemplo anterior, o número de divisores e a metragem de cabos foi maior, resultando em maior custo de implantação. Adicionalmente, esta topologia normalmente usa um cabo duplo no anel, o que aumenta ainda mais o custo da rede. Apesar destas desvantagens, as simulações mostraram que a utilização em conjunto com divisores assimétricos apresenta melhores margens de potência quando se tem um baixo número de ONUs na rede. No entanto, quando se usa divisores assimétricos o 
desempenho é mais sensível ao aumento da quantidade de ONUs do que a configuração em árvore.

- Topologia em barramento: A vantagem desta topologia é a economia de potência óptica, em casos onde os grupos de clientes estão muito distantes entre eles e da OLT. A topologia permite uma melhor gerência da rede e admite expansão futura. Além disso, foi a topologia que utilizou a menor metragem de cabos. Todavia, o número de divisores foi maior que a topologia em árvore.

- Topologia em árvore: Minimiza o número de divisores, devido ao fato deles serem simétricos, mas a metragem de cabos é maior que na topologia em barramento e igual ou menor que a topologia em anel.

\section{CONCLUSÕES}

A grande maioria das redes de acesso óptico do tipo PON sendo instaladas são baseadas na topologia em árvore e no uso de divisores de potência simétricos (razão de divisão 1:N), independente da tecnologia que estas empreguem, i.e., GPON ou EPON. Entretanto, considerando que os custos da implantação é fortemente afetado pelo custo do cabeamento da rede (em geral envolve pagamento de taxas de direito de passagem e obras civis), é importante que se disponha de ferramentas de planejamento visando otimizar a metragem de cabos ópticos instalados bem como o posicionamento dos divisores ópticos.

Neste artigo demonstramos que, dependendo da área geográfica a ser coberta, por exemplo, em aplicações onde as ONUs estão distribuídas de forma linear, o uso de divisores assimétricos, em combinação com divisores simétricos numa topologia em anel pode levar a uma melhoria na margem de potência média de cerca de $3,2 \mathrm{~dB}$. As vantagens do uso da topologia em anel podem ser estendidas para aspectos de proteção da rede, como demonstrado em [10]. Além disso, mesmo no caso da topologia em anel simétrica, a melhoria na margem em relação à árvore é de $1,3 \mathrm{~dB}$.
Em continuidade a este trabalho, usaremos seus resultados como referência para uma abordagem geral, com base em algoritmos genéticos. Desta forma, poderemos obter mecanismos para uma otimização do posicionamento e dimensionamento de redes PON que combinem as três topologias, por exemplo, árvore/barramento ou anel/árvore, com mais de um estágio de distribuição de potência.

\section{REFERENCIAS}

[1] .C. -H. Lee, W. V. Sorin, " Fiber to the Home Using a PON Infrastructure”, Journal of Lightwave Technology, V. 24, N. 12, pp. $4568-4583,2006$.

[2] .ITU-T G.984.1 (2003) - Gigabit-capable Passive Optical Networks (GPON): General characteristics

[3] .ITU-T G.984.2 (2003) - Gigabit-capable Passive Optical Networks (GPON): Physical Media Dependent (PMD) layer specification

[4] .ITU-T G.984.3 (2004) - Gigabit-capable Passive Optical Networks (GPON): Transmission convergence layer specification

[5] . ITU-T G.984.4 (2004) - Gigabit-capable Passive Optical Networks (GPON): ONT management and control interface specification

[6] .IEEE 802.3ah EPON Framework

[7] .B. Perkins, "Optimal Splitter Placement in PONs", Bechtel Telecommunications Technical Journal, V. 2, N. 2, pp. 49-52, 2004.

[8] B. Perkins, "Reducing the Amount of Fiber in Fiber-to-theHome Networks", Bechtel Telecommunications Technical Journal, V. 4, N. 1, pp. 1-8, 2006

[9] M.R. X. Barros, S.M. Rossi, A. E. Reggiani, C.A. Hortencio, J.G. D. Aguiar, D.C. Dini, P.C.L. Morais, M.L.C. Braga, M.R. Salvador, R. Bernardo, R.F. Silva, M.P. Mokarzel, R.A. Moreira, V. Corso, "Avaliação de Topologia para Redes GPON com Distribuição Assimétrica”, CPqD Cadernos de Tecnologia, Vol3, N.2, artigo 5, 2007

[10] Chien-Hung Yeh; Sien Chi, "Self-Healing Ring-Based TimeSharing Passive Optical Networks", IEEE Photonics Technology Letters, V.19, N.15, pp.1139 - 1141, 2007. 